\title{
BRANCHED CAUCHY-RIEMANN STRUCTURES ON ONCE-PUNCTURED TORUS BUNDLES
}

\author{
ALEX CASELLA
}

\author{
(Received 7 March 2019; first published online 17 May 2019)
}

2010 Mathematics subject classification: primary 57M50; secondary 32V05.

Keywords and phrases: geometric structures, Cauchy-Riemann, torus bundles, ideal triangulations, branching.

A geometry or geometric structure $(G, X)$ is a homogeneous space $X$ together with a transitive action on $X$ by a Lie group $G$, which acts as the symmetry group of the geometry. This concept was originally introduced by Klein in his celebrated Erlangen program and rapidly developed by Ehresmann and many others. When $X$ and $G$ are chosen appropriately, one recovers many classical geometries such as hyperbolic $\left(\mathrm{SO}(1, n), \mathbb{H}^{n}\right)$, Euclidean $\left(\mathbb{R}^{n} \rtimes \mathrm{O}(n), \mathbb{E}^{n}\right)$ or spherical $\left(\mathrm{O}(n+1), \mathbb{S}^{n}\right)$ geometry. A $(G, X)$-manifold $M$ is a manifold endowed with a $(G, X)$-structure, namely an atlas of charts in the model space $X$, whose transition functions are restrictions of elements of $G$. Associated to every $(G, X)$-structure is a developing map and holonomy representation

$$
\operatorname{dev}: \widetilde{M} \rightarrow X \quad \text { and } \quad \text { hol }: \pi_{1}(M) \rightarrow G
$$

such that

$$
\operatorname{hol}(\gamma) \cdot \operatorname{dev}(x)=\operatorname{dev}(\gamma \cdot x) \quad \text { for } \gamma \in \pi_{1}(M) \text { and } x \in \widetilde{M}
$$

The developing pair (dev, hol) is uniquely determined up to conjugation by an element of $G$, and so it is an invariant of the $(G, X)$-structure on $M$. Furthermore, the Ehresmann-Thurston principle implies that sufficiently nearby $(G, X)$-structures are completely determined by their holonomy representations. On the other hand, it is a rather complicated task to determine whether a representation $\rho: \pi_{1}(M) \rightarrow G$ arises as the holonomy of some $(G, X)$-structure.

As more and more connections between topology and geometry were discovered, $(G, X)$-structures have become a central topic in the study of manifolds (see, for example, [4, 7]). In a recent paper [7], Fock and Goncharov put together ideal

Thesis submitted to The University of Sydney in June 2018; degree approved on 28 August 2018; supervisor Stephan Tillmann, auxiliary supervisor Alexander Molev.

(C) 2019 Australian Mathematical Publishing Association Inc. 
triangulations and Thurston's ideas to fill in the gap between representations and geometry of surfaces. Given a surface $S$ and a group $G$, they use flags to parametrise decorated representations of $\pi_{1}(S)$ in $G$. Decorated representations are representations enriched with some geometry, which are only one step away from being $(G, X)$ structures. When $G=\operatorname{PGL}(3, \mathbb{R})$, they show that most of these representations can be promoted to holonomies of convex projective structures on $S$, geometric structures modelled on projective (PGL $\left.(3, \mathbb{R}), \mathbb{R P}^{2}\right)$ geometry [8]. Generalisations of these flag parametrisations to 3-manifolds are the Ptolemy coordinates of Garoufalidis, Thurston and Zickert [10] for $G=\operatorname{SL}(m, \mathbb{C})$, and the shape coordinates of Garoufalidis, Goerner and Zickert [9] for $G=\operatorname{PGL}(m, \mathbb{C})$. Some of these coordinate systems were independently developed by Bergeron, Falbel and Guilloux [2] for $G=\operatorname{PGL}(3, \mathbb{C})$, following the work of Falbel [5] on Cauchy-Riemann $\left(\mathbb{S}^{3}, \mathrm{PU}(2,1)\right)$ structures (CR for short).

In this thesis, we propose a different way to extend Fock and Goncharov's coordinate system to dimension three, for a special class of manifolds. The spaces we are interested in are punctured surface bundles, orientable manifolds which are the interior of compact 3-manifolds with boundary a union of tori. They are fibre bundles over the circle, with fibre space a punctured surface.

This thesis concentrates on the case where the surface is a once-punctured torus. The figure-eight knot complement is one such example. Most of these manifolds are hyperbolic and exhibit important combinatorial properties. In particular, Floyd and Hatcher showed that each hyperbolic once-punctured torus bundle admits a canonical realisation as an ideal triangulation, called the monodromy ideal triangulation [6]. The importance of this decomposition relies on its rich combinatorial structure, but also on its geometric properties. For instance, it was employed by Guéritaud [11], together with Casson's volume maximisation principle for angle structures, to prove hyperbolicity.

We show that a subset of the set of conjugacy classes of decorated representations is a subvariety of $(\mathbb{C} \backslash\{0,-1\})^{8}$, using the fact that the monodromy ideal triangulation is constructed by layering tetrahedra over a once-punctured torus. In particular, we give a concrete description of this algebraic variety in terms of fixed points of some explicit functions, called edge flips. This provides a coordinate system in eight complex variables of the character variety of a hyperbolic once-punctured torus bundle. Although related to the work in [2], our approach gives a different point of view. As an immediate consequence, we find that every hyperbolic once-punctured torus bundle has a special representation $\rho_{P}$, whose decorated character $\left[\rho_{P}\right]$ is shown to have special properties. The representation $\rho_{P}$ is irreducible and its image lies inside a subgroup of PGL(3, $\mathbb{C})$, sometimes called the Eisenstein-Picard modular group. It is the subgroup of $\mathrm{PU}(2,1)$ with entries in the ring of integers in the imaginary quadratic number field $\mathbb{Q}[\sqrt{-3}]$. In particular, this implies that the image of $\rho_{P}$ is discrete and one might wonder if it could be realised as the holonomy of a geometric structure.

In [5], Falbel finds the same representation $\rho_{P}$ in the case of the figure-eight knot complement $K_{8}$, the simplest hyperbolic once-punctured torus bundle. He shows that 
$\rho_{P}$ is the only representation of $\pi_{1}\left(K_{8}\right)$ in $\mathrm{PU}(2,1)$ whose restriction to the peripheral subgroup is faithful and purely parabolic. Moreover, he constructs a branched CR structure on $K_{8}$ whose holonomy is $\rho_{P}$. Such CR structures are modelled on the three-sphere $\mathbb{S}^{3} \subset \mathbb{C}^{2}$ together with the action of $\mathrm{PU}(2,1)$, its group of biholomorphic transformations. They are branched when the charts are locally branched coverings.

Inspired by the work of Falbel and Thurston, we modify the monodromy ideal triangulation of each once-punctured torus bundle to a new ideal cell decomposition. This decomposition is made up of tetrahedra and 3-cells that we call slabs, $\mathrm{CW}$ complexes obtained by deformation retracting the base of a square pyramid onto one of its sides. In the case of the figure-eight knot complement, Falbel uses one of these slabs implicitly, as part of a generalised tetrahedron. The CR structure thus constructed consists of charts that are not embeddings of the tetrahedra, and it does not generalise further. On the other hand, we geometrically realise each ideal cell by embedding it in CR space and use the malleability of slabs to build CR structures on almost all once-punctured torus bundles [3]. For this to work, six geometrically different types of slabs will be defined. A collection of the main results is summarised in the following theorem.

Theorem. Let $M_{f}$ be a hyperbolic once-punctured torus bundle. Then $M_{f}$ admits an ideal cell decomposition $\mathcal{D}_{f}$ that is geometrically realisable in $C R$ space. It corresponds to a branched CR structure, whose branch locus is the set of edges of $D_{f}$.

Moreover, the restriction of its associated decorated holonomy to the fundamental group $\langle\alpha, \beta\rangle$ of the base once-punctured torus does not depend on the monodromy automorphism $f$. It is the decorated character $\left[\rho_{P}, \Phi_{P}\right]$, where

$$
\rho_{P}(\alpha)=\left[\begin{array}{ccc}
\bar{\omega} & 0 & 0 \\
-1 & \omega & -\omega \\
\bar{\omega} & 0 & -1
\end{array}\right], \quad \rho_{P}(\beta)=\left[\begin{array}{ccc}
1 & -\omega & -1 \\
0 & \bar{\omega} & 0 \\
0 & \bar{\omega} & -\omega
\end{array}\right] \quad \text { and } \quad \omega=-\frac{1}{2}(1+\sqrt{-3})
$$

In particular, its Fock-Goncharov coordinates are

$$
\Psi_{f}\left(\left[\operatorname{hol}_{f}, \operatorname{dev}_{f}^{(0)}\right]\right)=(\omega, \omega, \omega, \omega, \omega, \omega, \omega, \omega) .
$$

In the end, we also analyse the branch locus and give simple descriptions of the ramification orders in terms of the combinatorics of the ideal cell decomposition.

The work done in this project has the potential to further extend to more general punctured surface bundles, as they also admit layered triangulations. Even though the number of coordinates (and the complexity of the problem) increases as the Euler characteristic of the punctured surface decreases, we do not see any theoretical barriers in the parametrisations of decorated characters. On the contrary, it is not clear whether one could construct $\mathrm{CR}$ structures in a similar way, as the new cell decompositions described here rely on the fact that the base surface is a once-punctured torus. We intend to address this problem in future work using the veering triangulations of Agol [1]. 


\section{References}

[1] I. Agol, 'Ideal triangulations of pseudo-Anosov mapping tori', in: Topology and Geometry in Dimension Three, Contemporary Mathematics, 560 (American Mathematical Society, Providence, RI, 2011), 1-17.

[2] N. Bergeron, E. Falbel and A. Guilloux, 'Tetrahedra of flags, volume and homology of SL(3)', Geom. Topol. 18(4) (2014), 1911-1971.

[3] A. Casella, 'Branched Cauchy-Riemann structures on once-punctured torus bundles', Geom. Topol., to appear.

[4] A. Casella, F. Luo and S. Tillmann, 'Pseudo-developing maps for ideal triangulations II: positively oriented ideal triangulations of cone-manifolds', Proc. Amer. Math. Soc. 145(8) (2017), 3543-3560.

[5] E. Falbel, 'A spherical CR structure on the complement of the figure eight knot with discrete holonomy', J. Differential Geom. 79(1) (2008), 69-110.

[6] W. Floyd and A. Hatcher, 'Incompressible surfaces in punctured-torus bundles', Topology Appl. 13(3) (1982), 263-282.

[7] V. Fock and A. Goncharov, 'Moduli spaces of local systems and higher Teichmüller theory', Publ. Math. Inst. Hautes Études Sci. 1(103) (2006), 1-211.

[8] V. V. Fock and A. B. Goncharov, 'Moduli spaces of convex projective structures on surfaces', Adv. Math. 208(1) (2007), 249-273.

[9] S. Garoufalidis, M. Goerner and C. K. Zickert, 'Gluing equations for PGL $(n, C)$-representations of 3-manifolds', Algebr. Geom. Topol. 15(1) (2015), 565-622.

[10] S. Garoufalidis, D. P. Thurston and C. K. Zickert, 'The complex volume of SL( $n, C)$ representations of 3-manifolds', Duke Math. J. 164(11) (2015), 2099-2160.

[11] F. Guéritaud, 'On canonical triangulations of once-punctured torus bundles and two-bridge link complements, with an appendix by David Futer', Geom. Topol. 10 (2006), 1239-1284.

\section{ALEX CASELLA, Department of Mathematics, Florida State University, Tallahassee, FL, USA \\ e-mail: acasella@fsu.edu}

